\title{
Understanding Users by Their D.I.S.C. Personality through Interactive Gaming
}

\author{
Qin En Looi ${ }^{1}$, Swee Lan See ${ }^{1}$, Chi Shien $\mathrm{Tay}^{2}$, and Gin Kee $\mathrm{Ng}^{2}$ \\ ${ }^{1}$ Institute for Infocomm Research, A*STAR, Singapore 138632 \\ ${ }^{2}$ Catholic High School, Singapore 579767 \\ \{Qin En Looi, Swee Lan See, Chi Shien Tay, \\ Gin Kee Ng, stuqel\}@i2r.a-star.edu.sg
}

\begin{abstract}
A key component of improving human-computer interaction is through the matching of users with their preferred computer interfaces and interaction styles. Understanding the users better would result in a customized gaming experience, leading to sustained user engagement. In this paper, we develop an alternative tool to aid in the measurement of the D.I.S.C. personality styles of users in the form of an interactive game. Through this game, we aim to predict the personality type of the gamer, from which invaluable insights about each type of gamer can be elicited.
\end{abstract}

Keywords: Alternative assessment tool, D.I.S.C. Personality Profiling, Interactive Gaming.

\section{Introduction}

With this growing need and pervasive use of information technologies, the study of Human-Computer Interaction (HCI) is undoubtedly becoming more and more important. Some studies have identified that the choice of computer interface and interaction style needs to match the users to result in good human computer interaction. Hence, it would be beneficial to understand users better and hence develop computer interfaces that could serve to improving human computer interactions.

We hypothesised that interactive social gaming could reveal the gamers' personality traits through the in-game decisions and activities. Hence, we decided to conduct further studies in this aspect of attempting to understand the users better through their D.I.S.C. personality traits.

In this paper, we would discuss about eliciting insights from user behaviours through the development an interactive game, followed by human behaviour assessment methods, which leads to the generic framework towards understanding users when developing an interactive game.

\section{Personality Assessments}

Personality is an important aspect as it shapes a human's mind and thoughts. Many personality assessment methods were developed and used by psychologist and 
researchers. Basically, there are two major types of personality assessments, namely: Projective tests and Objective tests.

Projective tests assume that personality is primarily unconscious and assess an individual by how he or she responds to an ambiguous stimulus, like an ink blot. The idea is unconscious needs will come out in the person's response, e.g. an aggressive person may see images of destruction. On the other hand, objective tests assume personality is consciously accessible and measure it by self-report questionnaires.

Research on psychological assessment has generally found objective tests to be more valid and reliable than projective tests. Some of the notable objective tests include personality assessments such as the DISC personality profiling, the MyersBriggs Type Indicator, Holland Codes, Minnesota Multiphasic Personality Inventory and many more.

Personality assessments can be scored using a dimensional (normative), a typological (ipsative) approach or both. Dimensional approaches, such as the Big 5, describe personality as a set of continuous dimensions on which individuals differ. On the other hand, typological approaches, such as the Myers-Briggs Type Indicator, describe opposing categories of functioning where individuals differ. Normative responses for each category can be graphed as bell curves (normal curves), implying that some aspects of personality are better than others. Ipsative test responses offer two equally "good" responses between which an individual must choose. Such responses (e.g., on the MBTI) would result in bi-modal graphs for each category, rather than bell curves. Personality tests, such as the Strength Deployment Inventory (r), which assesses motivation, or purpose, of behaviour, rather than the behaviour itself, combine a dimensional and typological approach. Three continuums of motivation are combined to yield 7 distinct types.

These personality assessments allow people to understand their way of thinking and how they react to certain situations. Through these personality assessments, researchers in the field of HCI are able to shape different programmes and ideas to suit different users of different personalities and hence, achieve the basic goal in the study of HCI. In addition, users who understand their personalities are able to understand their strengths and weaknesses as well, so that they are able to make better achievements in their career and life.

In this paper, we are attempting to improve understanding of people through the use of a personality assessment known as D.I.S.C personality profiling, which is similar to BIG FIVE, and at the same time through developing an interactive game. We use this personality assessment to extend the observation found in social gaming. D.I.S.C is a personality assessment developed based on the 1928 work of psychologist William Moulton Marston and the original behaviouralist Walter V. Clarke, et al. $D$ represents Dominance (relating to control, power and assertiveness), $I$ represents Influence (relating to social situations and communication), $S$ represents Steadiness (relating to patience, persistence and thoughtfulness), and $C$ represents Conscientiousness (relating to structure and organization). 


\section{Results and Discussion}

The Preliminary Results were collected through the means of a quantitative questionnaire and the results are as shown in the figures below. $70 \%$ of the sample group have 'I' \& 'S' personality types and almost all 'I' \& 'S' personalities play computer games (Fig. 1), and form a potential gaming market, while ' $\mathrm{D}$ ' \& 'C' personalities are less engaged in computer games possibly due to their critical nature.

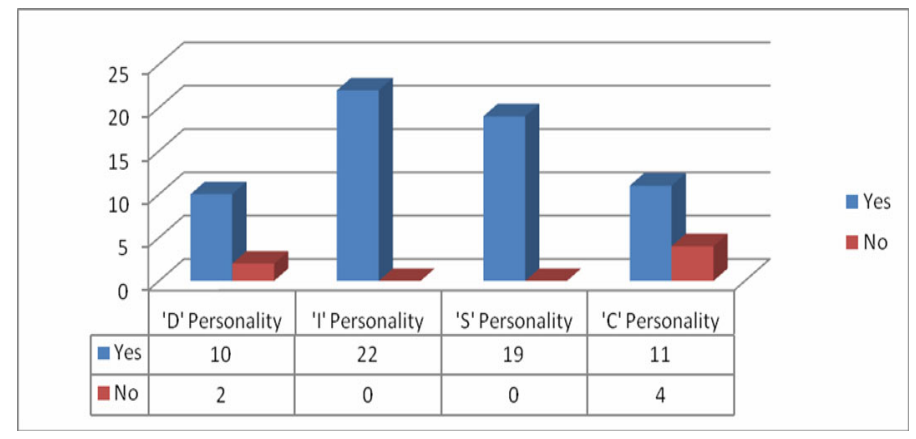

Fig. 1. Distribution of Personality Types in Gamers and Non-Gamers

Fig. 2 shows people of ' $\mathrm{S}$ ' personality has the highest demand for a good quality game, for instance, games should meet standards such as having specific mentioned features in the games they play, even though this question asked them about their favorite game. Fig. 3 also shows that teenagers largely prefer multi-player games and interactive, role-playing games compared to less popular genres such as educational games In addition, from Table 1, we can further narrow down to people with ' $\mathrm{D}$ ' and ' $\mathrm{S}$ ' personalities being more demanding in their preference of their ideal game.

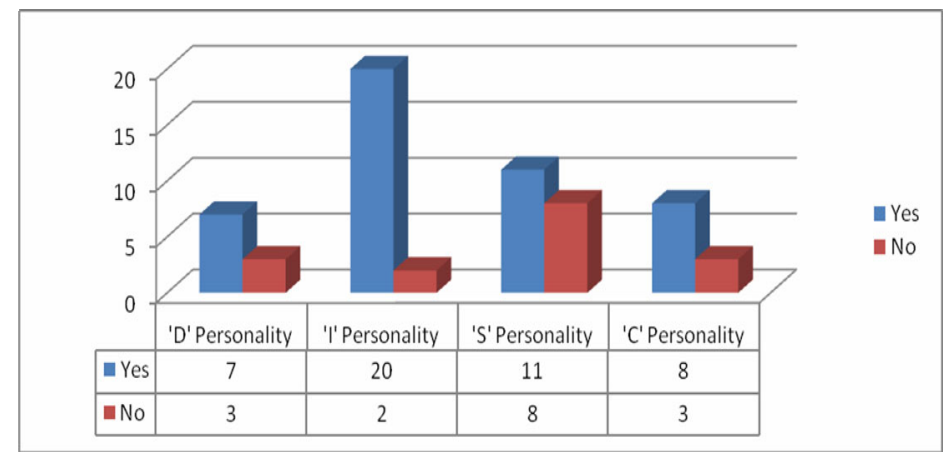

Fig. 2. Distribution of Personality Types and Favorite Game Expectations 


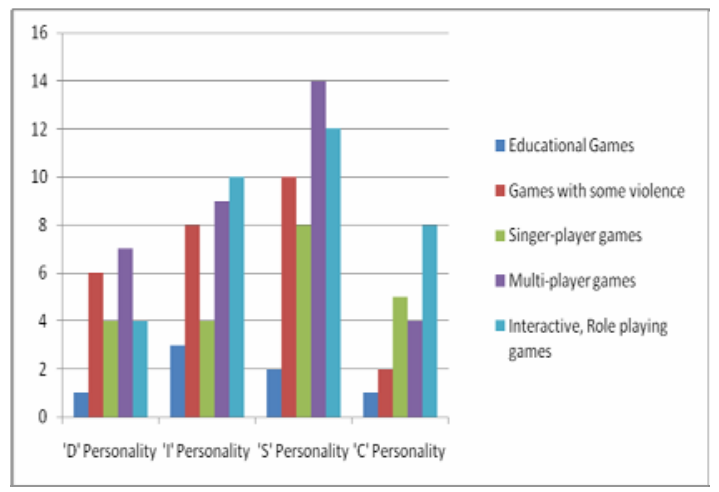

Fig. 3. Distribution of Personality Types and Favorite Game Expectations

Table 1. Personality Types and Ideal Game Characteristics

\begin{tabular}{lcccc}
\hline Personalities/ & D & I & S & C \\
Characteristics & & & & \\
\hline Good Graphics & $\checkmark$ & $\checkmark$ & $\checkmark$ & \\
3 Dimensional & $\checkmark$ & & $\checkmark$ & $\checkmark$ \\
Good strategy & $\checkmark$ & & $\checkmark$ & \\
Interactive & & $\checkmark$ & & \\
Able to Win & $\checkmark$ & & & \\
Realistic & & & $\checkmark$ & \\
\hline
\end{tabular}

From Table 2, we can see that certain features are only selected by certain D.I.S.C. personality types. For example, 'D' personalities prefer using console; 'I' personalities prefer playing speech-controlled games; ' $S$ ' personalities prefer playing first person shooter games while ' $C$ ' personalities showed no interest to these three features. This shows that people of each D.I.S.C. personality type do display distinct gamer behavior when playing games. Therefore, we are able to confirm with our hypothesis that gamers display their D.I.S.C. behavioral traits in the course of game play.

Table 2. Personality Types and Game Features Selections

\begin{tabular}{lcccc}
\hline Personalities/ & D & I & S & C \\
Features & & & & \\
\hline Gesture & $\checkmark$ & $\checkmark$ & $\checkmark$ & $\checkmark$ \\
Use of Keyboard & & $\checkmark$ & $\checkmark$ & $\checkmark$ \\
3 Dimensional & & & $\checkmark$ & $\checkmark$ \\
Graphics & & & $\checkmark$ & \\
Real Life Simulation & $\checkmark$ & & $\checkmark$ & \\
Speech Control & & $\checkmark$ & & \\
Console & $\checkmark$ & & & \\
\hline
\end{tabular}

Results from the Preliminary Questionnaire shows that the expectation level of a game varies with gamers of different D.I.S.C. personality types. ' $D$ ' and ' $C$ ' personality gamers, who dislike routine, and being more critical in nature, are 
generally found to be less engaged in online gaming. On the other hand, ' $\mathrm{I}$ ' and ' $\mathrm{S}$ ' personality gamers, who are generally interactive, and enjoy simple pleasures, are found to be more engaged in online games.

\section{Conclusions}

From this research, we found that users of ' $S$ ' personality style are better reviewers compared to users of other personality types. Game developers who would like to create better interaction interfaces should consult them for their inputs. However, as this is a preliminary study with small sample size, our future research would attempt to verify to confirm this finding.

In conclusion, from social gaming, we could understand the users better and can observe a user's personality. Through understanding users by their D.I.S.C. personality style, we could create better gaming interfaces to improving human computer interactions, thus enhancing user experience.

\section{References}

1. See, S.L., Tan, M., Looi, Q.E.: Towards better human robot interaction: Understand human computer interaction in social gaming using a video-enhanced diary method. In: Kim, J.-H., Ge, S.S., Vadakkepat, P., Jesse, N., Al Manum, A., Puthusserypady, K.S., Rückert, U., Sitte, J., Witkowski, U., Nakatsu, R., Braunl, T., Baltes, J., Anderson, J., Wong, C.-C., Verner, I., Ahlgren, D. (eds.) Progress in Robotics. CCIS, vol. 44, pp. 119-127. Springer, Heidelberg (2009)

2. Kim, J.-H., Ge, S.S., Vadakkepat, P.: Advances in Robotics. LNCS, vol. 5744. Springer, Heidelberg (2009)

3. Tan, M., Looi, Q.E., See, S.L.: Social Gaming: What Attracts the Most Attention? An Investigation Using an Improved Diary Method. In: Proceedings of the International Conference for Advances in Computer Entertainment Technology, p. 415 (2008)

4. Viswesvaran, C., Ones, D.S.: Meta-Analyses of Fakability Estimates: Implications for Personality Measurement. Educational and Psychological Measurement 59(2), 197-210 (1999) 